\title{
The association of maternal vitamin D status with infant birth outcomes, postnatal growth and adiposity in the first 2 years of life in a multi-ethnic Asian population: the Growing Up in Singapore Towards healthy Outcomes (GUSTO) cohort study
}

Yi Lin Ong ${ }^{1} \dagger$, Phaik Ling Quah ${ }^{1} \dagger$, Mya Thway Tint ${ }^{2}$, Izzuddin M. Aris ${ }^{3}$, Ling Wei Chen ${ }^{3}$, Rob M. van Dam ${ }^{4}$, Denise Heppe ${ }^{5}$, Seang-Mei Saw ${ }^{4}$, Keith M. Godfrey ${ }^{6}$, Peter D. Gluckman ${ }^{1,7}$, Yap Seng Chong ${ }^{1,2}$, Fabian Yap ${ }^{8,9}$, Yung Seng Lee ${ }^{1,3,10}$ and Mary Foong-Fong Chong ${ }^{1,3,10 *}$ on behalf of the GUSTO group ${ }^{1}$ Singapore Institute for Clinical Sciences, Agency for Science, Technology and Research, Brenner Centre for Molecular Medicine, 30 Medical Drive, Singapore 117609, Singapore

${ }^{2}$ Department of Obstetrics and Gynaecology, Yong Loo Lin School of Medicine, National University of Singapore and National University Health System, Tower Block, Level 12, 1E Kent Ridge Road, Singapore 119228, Singapore

${ }^{3}$ Department of Paediatrics, Yong Loo Lin School of Medicine, National University of Singapore and National University Health System, Tower Block, Level 12, 1E Kent Ridge Road, Singapore 119228, Singapore

${ }^{4}$ Saw Swee Hock School of Public Health, National University of Singapore, Tahir Foundation Building, 12 Science Drive 2 \#10-01, Singapore 117549, Singapore

${ }^{5}$ Department of Pediatrics and of Epidemiology, Erasmus Medical Center, 's-Gravendijkwal 230, 3015 CE Rotterdam, The Netherlands

${ }^{6}$ MRC Lifecourse Epidemiology Unit, NIHR Southampton Biomedical Research Centre, University of Southampton and University Hospital Southampton NHS Foundation Trust, MP 218, Tremona Road, Southampton General Hospital, Southampton SO16 6YD, UK

${ }^{7}$ Liggins Institute, University of Auckland, 85 Park Avenue, Grafton, Auckland 1023, New Zealand

${ }^{8}$ Duke-NUS Graduate Medical School, 8 College Road, Singapore 169857, Singapore

${ }^{9}$ Department of Pediatrics, KK Women's and Children's Hospital, 100 Bukit Timah Road, Singapore 229899, Singapore

${ }^{10}$ Clinical Nutrition Research Center, Singapore Institute for Clinical Sciences (SICS), Agency for Science, Technology and Research (A*STAR), Medical Drive \#07-02, MD 6 Building, Yong Loo Lin School of Medicine, Singapore 117599, Singapore

(Submitted 21 September 2015 - Final revision received 24 January 2016 - Accepted 26 January 2016 - First published online 24 June 2016)

\section{Abstract}

Maternal vitamin D status during pregnancy has been associated with infant birth and postnatal growth outcomes, but reported findings have been inconsistent, especially in relation to postnatal growth and adiposity outcomes. In a mother-offspring cohort in Singapore, maternal plasma vitamin D was measured between 26 and 28 weeks of gestation, and anthropometric measurements were obtained from singleton offspring during the first 2 years of life with 3-month follow-up intervals to examine birth, growth and adiposity outcomes. Associations were analysed using multivariable linear regression. Of a total of 910 mothers, $13.2 \%$ were vitamin $\mathrm{D}$ deficient $(<50 \mathrm{nmol} / \mathrm{l})$ and $26.5 \%$ were insufficient $(50-75 \mathrm{nmol} / \mathrm{l})$. After adjustment for potential confounders and multiple testing, no statistically significant associations were observed between maternal vitamin D status and any of the birth outcomes - small for gestational age (OR 1.00; 95\% CI 0.56, 1.79) and pre-term birth (OR 1·16; $95 \%$ CI $0 \cdot 64,2 \cdot 11$ ) - growth outcomes - weight-for-age $z$-scores, length-for-age $z$-scores, circumferences of the head, abdomen and mid-arm at birth or postnatally - and adiposity outcomes - BMI, and skinfold thickness (triceps, biceps and subscapular) at birth or postnatally. Maternal vitamin D status in pregnancy did not influence infant birth outcomes, postnatal growth and adiposity outcomes in this cohort, perhaps due to the low prevalence ( $1.6 \%$ of the cohort) of severe maternal vitamin D deficiency (defined as of $<30 \cdot 0 \mathrm{nmol} / \mathrm{l})$ in our population.

Key words: Maternal vitamin D: Birth outcomes: Growth outcomes: Infant anthropometric measures: Adiposity outcomes: Pregnancy

Abbreviations: 25(OH)D, 25-hydroxyvitamin D; BMIZ, BMI-for-age $z$-scores; LAZ, length-for-age z-scores; SGA, small for gestational age; WAZ, weight-for-age z-scores.

* Corresponding author: Dr M. F.-F. Chong, fax +656776 6840, email mary_chong@sics.a-star.edu.sg

$\dagger$ Both authors contributed equally to this work. 
Maternal nutrition during pregnancy is crucial for creating an optimal intra-uterine environment for fetal development ${ }^{(1)}$ and has been linked to subsequent (postnatal) growth ${ }^{(2-4)}$. The role of vitamin D in pregnancy is of particular interest, with reported associations between vitamin $\mathrm{D}$ deficiency in pregnant woman and poor offspring fetal and postnatal growth ${ }^{(4-7)}$

With respect to birth outcomes, several observational studies have shown associations between maternal vitamin D deficiency (definitions in studies range from $<30$ to $37.5 \mathrm{nmol} / \mathrm{l}$ ), or lower maternal vitamin $\mathrm{D}$ concentrations with higher risk of small-for-gestational-age (SGA) infants ${ }^{(5-10)}$, pre-term births $^{(10)}$ and reduced birth weight, length, BMI and head circumference $^{(5,8,11,12)}$. In contrast, studies with higher vitamin D deficiency cut-offs - for example, $<50,>50,<80 \mathrm{nmol} / \mathrm{l}^{(13-16)}$ or relatively high mean population level of maternal vitamin $\mathrm{D}$ $(70 \mathrm{nmol} / \mathrm{l})^{(17)}$ - reported lack of associations with SGA ${ }^{(16,17)}$ and birth anthropometric measurements ${ }^{(13-15,17)}$. Taken together, these results show that vitamin $\mathrm{D}$ supplementation during pregnancy did not affect birth weight or birth length, which are in line with a Cochrane review of data from three trials ${ }^{(10)}$.

Evidences on maternal vitamin D and early postnatal growth outcomes are, however, less clear. When defined as $<30 \mathrm{nmol} / \mathrm{l}$, maternal vitamin $\mathrm{D}$ deficiency was associated with greater weight for age in the 1 st year of life ${ }^{(5,11)}$. Findings on length for age were mixed, with maternal deficiency being associated with lower length for age and smaller head circumference in one study $^{(11)}$, but higher length-for-age in another ${ }^{(5)}$. Mixed findings were also observed in studies where maternal vitamin $\mathrm{D}$ deficiency/insufficiency was defined at levels $>30 \mathrm{nmol} / \mathrm{l}^{(7,15,17)}$. Thus far, current findings relating maternal vitamin D to early postnatal growth outcomes are inconclusive.

There is growing evidence to suggest the role of maternal vitamin $\mathrm{D}$ in infant adiposity. However, findings are also conflicting, with two studies demonstrating no association between maternal vitamin D deficiency and infant skinfold measures $^{(13,14)}$ and one other study finding contradictory associations $^{(18)}$. Maternal vitamin D deficiency was associated with increased infant overweight risk at 1 year of age but not at 4 years $^{(19)}$, whereas in a separate study ${ }^{(20)}$ lower maternal vitamin D was associated with lower fat mass in the offspring at birth but greater fat mass at ages 4 and 6 years.

In summary, the current body of work suggests that the risks of pre-term birth, SGA and smaller birth sizes are less evident when pregnant women are deficient or insufficient in vitamin D defined as $>37.5-75 \mathrm{nmol} / \mathrm{l}$. However, the associations of maternal vitamin D deficiency with early postnatal growth outcomes and infant adiposity are less clear, with inconsistent directionality and a smaller number of studies.

We aimed to further elucidate the associations of maternal vitamin D status with infant birth outcomes, early postnatal growth and adiposity outcomes up to 2 years of age in a multi-ethnic Asian mother-offspring cohort in Singapore.

\section{Methods}

\section{Study design}

Data were obtained from the Growing Up in Singapore Towards healthy Outcomes (GUSTO) study, a population-based prospective cohort study in Singapore. Further details on the GUSTO study have been previously described by Soh et $a l^{(21)}$. Pregnant women ( $n$ 1247) within the age range of 18-50 years were recruited from the two major public maternity units in National University Hospital (NUH) and KK Women's and Children's Hospital (KKH). Participants had to be Singaporean citizens or permanent residents, of Chinese, Malay or Indian ethnicity with parents of homogeneous ethnic background, have the intention to deliver in $\mathrm{NUH}$ or $\mathrm{KKH}$, plan to reside in Singapore in the upcoming 5 years and had to be willing to donate birth tissue at delivery (cord, placenta and cord blood). Pregnant women on chemotherapy or with pre-existing health conditions including type 1 diabetes mellitus, depression or other mental health-related disorders were excluded from the study. Written informed consent was collected from all participants upon recruitment. This study was granted ethics approval by the Institutional Review Boards of the respective hospitals involved (clinical trial registry NCT01174875).

\section{Maternal and infant characteristics}

Demographic data on maternal age, ethnicity, educational level and smoking status as well as obstetric and medical history data were collected from the women at 26-28 weeks of gestation. Total maternal energy intake was ascertained from 24-h dietary recalls conducted at 26-28 weeks of gestation, and 3-d food diaries were also collected for validation purposes. Information on infant sex and birth order was obtained from birth delivery reports. Low birth weight was defined as $<2500$ g, and pre-term birth was defined as live birth before 37 weeks of gestation. Gestational age was determined from a dating ultrasound scan conducted in the first trimester. SGA was defined as birth weight $<10$ th percentile for gestational age using a birth weight reference developed from 19634 infants delivered at NUH, Singapore ${ }^{(22)}$.

\section{Maternal vitamin D assessment}

Maternal plasma samples collected at 26-28 weeks of gestation were analysed for serum vitamin $\mathrm{D}$ concentrations. The medians and interquartile ranges of the gestational ages are presented in Table 1. Extraction of 25-hydroxyvitamin $\mathrm{D}_{2}$ and $\mathrm{D}_{3}\left(25(\mathrm{OH}) \mathrm{D}_{2}\right.$ and $\left.\mathrm{D}_{3}\right)$ metabolites with hexane (Chromonorm) was conducted. Plasma samples were vortex-mixed with hexane to extract $25(\mathrm{OH}) \mathrm{D}_{2}$ and $\mathrm{D}_{3}$ metabolites, after which the hexane layer was evaporated to dryness and reconstituted using methanol-water (70:30 by volume) mixture (HyperSolv). Analysis of vitamin D concentration was conducted using liquid chromatography-tandem MS (Applied Biosystems) with Analyst ${ }^{\mathrm{TM}}$ software (version 1.3; Applied Biosystems). Chromatographic separation was carried out with a BDS C8 reversed-phase column (ThermoHypersil). Extraction and assessment methods have been described in greater detail previously ${ }^{(23)}$. The intra- and inter-assay $\mathrm{CV}$ for $25(\mathrm{OH}) \mathrm{D}_{2}$ and $25(\mathrm{OH}) \mathrm{D}_{3}$ were $\leq 10 \cdot 3 \%$, and the detection limit was $<4 \mathrm{nmol} / \mathrm{l}$ for both metabolites.

Singapore's $\left(1^{\circ} 22^{\prime} \mathrm{N}\right)$ climate, which is sun-rich all year round, is classified as tropical rainforest climate with no true distinct seasons; thus, there should be little impact of potential seasonal 
variation on 25-hydroxyvitamin D (25(OH)D) levels (Singapore Department of Statistics, 2014; http://www.singstat.gov.sg/).

\section{Infant anthropometric data}

Measurements of infant anthropometry were adopted from standardised protocols ${ }^{(24)}$. For most measurements (except skinfolds), duplicate measures were obtained (skinfolds in triplicates) and averaged. Infant birth weight was obtained from birth delivery reports, and birth length was measured within $72 \mathrm{~h}$ after birth using a mobile measuring mat (SECA model 210; SECA Corp.), recorded to the nearest $5 \mathrm{~mm}$. Infant weight at 3, $6,9,12,15$ and 18 months of age was measured to the nearest gram (SECA model 334; Seca Corp.). Toddler weight at 24 months of age was measured to the nearest kilogram using calibrated scales (SECA model 813; SECA Corp.). Between 3 and 18 months of age, recumbent infant length was measured from the top of the infant's head to the soles of the feet using an infant mat (SECA 210 mobile measuring mat), whereas standing height at 24 months of age was measured using a stadiometer (SECA 213 Mobile Stadiometer; SECA Corp.). Length and height (only at 24 months) measurements were obtained to the scale of $0 \cdot 1 \mathrm{~cm}$ and averaged from duplicate values.

Head circumference, abdominal circumference and mid-arm circumference measurements were similarly taken at birth and at 3, 6, 9, 12, 15, 18 and 24 months of age. Maximum head circumference was measured across the frontal bones of the skull and over the occipital prominence at the back of the head. Abdominal circumference was taken at the end of the infant's normal expiration. Mid-arm circumference was measured at half the distance between the acromion process and the olecranon. All measurements were recorded using a non-stretchable measuring band (SECA 212 Measuring Tape; SECA Corp.) to the nearest $0 \cdot 1 \mathrm{~cm}$.

Skinfold measurements of the triceps and subscapular regions were taken at birth and at 18 and 24 months of age, whereas measurements at the biceps were taken at 18 and 24 months of age. Skinfold measurements were taken in triplicates using Holtain skinfold calipers (Holtain Ltd) on the right side of the body, recorded to the nearest $0.2 \mathrm{~mm}$.

Anthropometric training and standardisation sessions were conducted quarterly (once every 3 months), and observers were trained to obtain anthropometric measurements that, on average, were closest to the values measured by a master anthropometrist. Assessment of reliability was estimated by inter-observer technical error of measurement and $\mathrm{CV}^{(25)}$ (online Supplementary Table S1).

\section{Statistical analysis}

From 1247 mothers, 127 (10.2\%) were excluded based on the following exclusion criteria: study dropouts, neonatal complications, twin birth and in vitro fertilisation (IVF) birth. Twins and IVF infants were excluded because of different growth trajectories compared with singleton, naturally conceived infants. From the remaining 1120 mothers, 910 (81.2\%) had blood plasma samples available for measurement of maternal vitamin D concentrations.
These mothers and their infants were included in the analysis of infant SGA and pre-term birth outcomes.

In the analysis for infant birth and postnatal growth outcomes, pre-term delivery or low birth weight ( $n$ 103) infants were excluded, as they were expected to have different growth trajectories compared with term infants with normal birth weights. The remaining 807 subjects with complete measures were included in the analysis. Owing to losses from follow-up, the number of subjects with available infant outcome measurement data at each time point varied, as reported in further detail in Fig. 1.

Maternal vitamin D status was defined as deficient $(<50 \mathrm{nmol} / \mathrm{l})$, insufficient $(\geq 50 \mathrm{nmol} / \mathrm{l}$ and $<75 \mathrm{nmol} / \mathrm{l})$ and sufficient $(\geq 75 \mathrm{nmol} / \mathrm{l})$ based on recommendations by the Endocrine Society Clinical Practice Guidelines ${ }^{(26)}$. The Institute of Medicine (IOM) guidelines were not used as they define severe vitamin D deficiency as vitamin D levels $<30 \mathrm{nmol} / \mathrm{l}$, of which we had very low numbers $(1.6 \%)$ in our cohort to provide sufficient power for analyses. The distribution of maternal and infant characteristics between maternal vitamin D categories was compared using the $\chi^{2}$ test for categorical variables and ANOVA for continuous variables. Frequencies of missing demographic data were low $(<5 \%)$, and hence imputed to retain good population representation. Data were assumed to be missing at random. Categorical variables were imputed as categories with the highest observed frequency in the study cohort. Continuous variables were imputed with the median values of the study cohort. Statistical significance for the descriptive data was defined as $P$ value $<0 \cdot 05$.

From the anthropometric data, age and sex-adjusted $z$-scores for weight, length and BMI measurements were derived with reference to the WHO 2006 standards for child growth ${ }^{(27)}$. Infant measures of linear growth included weight-for-age $z$-scores (WAZ) and length-for-age $z$-scores (LAZ) and head, abdominal and mid-arm circumferences. Adiposity outcomes included BMI-for-age $z$-scores (BMIZ) and skinfold measurement from the triceps, biceps and the subscapular region. Conditional changes in WAZ, LAZ and BMIZ across different time points at 3-month intervals up to 24 months of age were used as measures for gain in weight, length and BMI, respectively. $Z$-scores at each successive time point, conditional on the previous $z$-score, was calculated by saving the residuals from linear regression models of $z$-scores at each successive time point $v$. $z$-score at the earlier time point ${ }^{(28)}$. Therefore, the use of conditional change in $z$-scores between time points is indicative of growth within the specified time period, while accounting for outcomes at earlier time points.

For binary birth outcomes of SGA and pre-term birth, logistic regression analysis was used to examine the associations with maternal vitamin D status, adjusted for maternal ethnicity, education, smoking during pregnancy, age, pregnancy BMI, total maternal energy intake, infant birth order and infant sex as potential confounders. These confounders were selected if they were significantly associated with $25(\mathrm{OH}) \mathrm{D}$ levels or growth outcomes in univariate analyses, or reported in the literature.

Separate multivariable linear regression models were used to investigate associations between maternal vitamin D status 


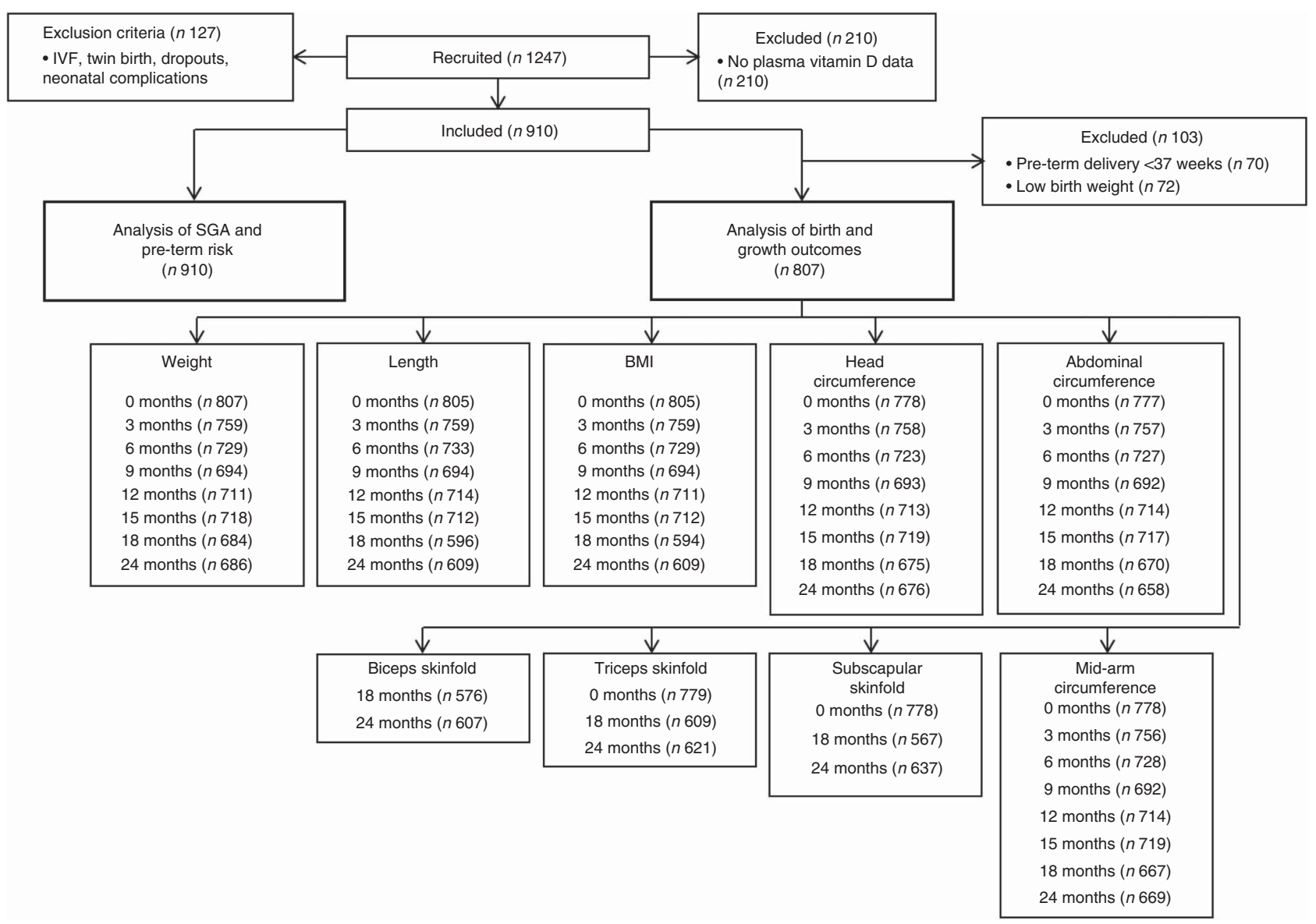

Fig. 1. Flowchart of study participants with data on birth outcomes and anthropometric measurements at various time points from birth to 24 months of age. IVF, in vitro fertilisation; SGA, small for gestational age.

during pregnancy and infant birth outcomes, postnatal growth and adiposity outcomes. For outcome variables normalised as $z$-scores (WAZ, LAZ, BMIZ, conditional change in $z$-scores), the regression model used was adjusted for the same potential confounders in the logistic regression model but without infant sex. For other infant outcome variables (head, mid-arm and abdominal circumferences, skinfold measurements), the regression model used was adjusted for the same potential confounders in the logistic regression model.

A sensitivity analysis was conducted using a subset of infants who had growth and adiposity measurements available at all time points between 0 and 24 months of age ( $n$ 399). This additional analysis was conducted to test the robustness of the findings and the same multivariable linear regression models were used in the analysis.

Statistical analyses were performed using standard statistical software (SPSS version 16.0; SPSS Inc.). As the postnatal growth and adiposity outcomes were taken at multiple time points, the Bonferroni correction was used to set a statistical significance for the $P$ value, to account for type I error due to multiple comparisons. Statistical significance in regression models was identified by a $P$ value of $<0 \cdot 006$, determined by accounting for the maximum 8 time points of measurement for infant outcome variables.

\section{Results}

\section{Characteristics of participants}

The maternal and infant characteristics of the study population $(n$ 910) are summarised in Table 1 . The mean maternal vitamin D concentration was 81.3 (SD $27 \cdot 2$ ) nmol/l, with $13 \cdot 2 \%$ of the population being vitamin $\mathrm{D}$ deficient and $26.5 \%$ being vitamin D insufficient. Compared with mothers who were sufficient in vitamin D, deficient or insufficient mothers tended to be of Indian and Malay instead of Chinese ethnicity, were younger, had lower educational levels and had lower pregnancy BMI. The percentage of mothers who were taking vitamin D supplementation was the lowest in those who were deficient $(63.9 \%)$, compared with those who were insufficient (80.6\%) and sufficient (86.3\%). Only $1.6 \%$ of mothers had severe vitamin D deficiency (defined as $<30.0 \mathrm{nmol} / \mathrm{l}$ by the IOM guidelines). No significant differences in all the maternal or infant characteristics were observed in the cohort of 807 subjects and the study subset of 399 subjects (online Supplementary Table S2).

Birth outcomes. Compared with mothers with sufficient vitamin $\mathrm{D}$, the likelihood of having a SGA baby or a pre-term birth was not significantly different from mothers with deficient 
Table 1. Comparison of maternal and infant characteristics of the study population ( $n$ 910) between maternal vitamin $\mathrm{D}$ categories

(Numbers and percentages for categorical variables or mean values and standard deviations for continuous variables; medians and interquartile ranges (IQR))

\begin{tabular}{|c|c|c|c|c|c|c|c|c|c|}
\hline & \multicolumn{2}{|c|}{ Overall ( $n$ 910) } & \multicolumn{2}{|c|}{ Deficiency $(<50 \mathrm{nmol} / \mathrm{l})(n 120)$} & \multicolumn{2}{|c|}{ Insufficiency ( $\geq 50$ and $<75 \mathrm{nmol} / \mathrm{l}$ ) ( $n$ 241) } & \multicolumn{2}{|c|}{ Sufficiency $(\geq 75 \mathrm{nmol} / \mathrm{l})(n 549)$} & \multirow[b]{2}{*}{$P$} \\
\hline & Mean $/ n$ & $\mathrm{SD} / \%$ & Mean $/ n$ & $\mathrm{SD} / \%$ & Mean $/ n$ & $\mathrm{sD} / \%$ & Mean $/ n$ & $\mathrm{sD} / \%$ & \\
\hline \multicolumn{10}{|l|}{ Maternal characteristics } \\
\hline Maternal vitamin D plasma concentration (nmol/l) & $81 \cdot 3$ & $27 \cdot 2$ & 39.0 & 7.5 & $63 \cdot 0$ & $7 \cdot 1$ & 98.7 & $18 \cdot 6$ & $<0.001$ \\
\hline Maternal age (years) & 30.5 & $5 \cdot 1$ & $29 \cdot 0$ & $5 \cdot 3$ & $29 \cdot 7$ & 5.0 & 31.3 & 5.0 & $<0.001$ \\
\hline Maternal BMl at 26 weeks $\left(\mathrm{kg} / \mathrm{m}^{2}\right)^{*}$ & $26 \cdot 1$ & 4.3 & $26 \cdot 8$ & 4.5 & $26 \cdot 4$ & 4.7 & $25 \cdot 8$ & $4 \cdot 1$ & 0.033 \\
\hline Total maternal energy intake (kJ) & 8489 & 5389 & 9045 & 7920 & 8267 & 4937 & 8460 & 4874 & 0.432 \\
\hline Total maternal energy intake (kcal) & 2029 & 1288 & 2161 & 1893 & 1976 & 1180 & 2022 & 1165 & 0.432 \\
\hline Ethnicity & & & & & & & & & $<0.001$ \\
\hline Chinese & 509 & $55 \cdot 9$ & 27 & 22.5 & 96 & 39.8 & 386 & $70 \cdot 3$ & \\
\hline Malay & 232 & $25 \cdot 5$ & 56 & $46 \cdot 7$ & 87 & $36 \cdot 1$ & 89 & $16 \cdot 2$ & \\
\hline Indian & 169 & 18.6 & 37 & 30.8 & 58 & $24 \cdot 1$ & 74 & 13.5 & \\
\hline Education* & & & & & & & & & 0.003 \\
\hline Primary and secondary & 275 & $30 \cdot 2$ & 44 & $36 \cdot 7$ & 64 & $26 \cdot 6$ & 167 & 30.4 & \\
\hline Post-secondary & 322 & 35.4 & 42 & $35 \cdot 0$ & 107 & 44.4 & 173 & 31.5 & \\
\hline University & 313 & 34.4 & 34 & $28 \cdot 3$ & 70 & 29.0 & 209 & 38.1 & \\
\hline Smoked regularly during pregnancy* & & & & & & & & & 0.287 \\
\hline No & 886 & $97 \cdot 4$ & 116 & $96 \cdot 7$ & 238 & 98.8 & 532 & $96 \cdot 9$ & \\
\hline Yes & 24 & 2.6 & 4 & 3.3 & 3 & 1.2 & 17 & $3 \cdot 1$ & \\
\hline \multicolumn{10}{|l|}{ Vitamin D supplements during pregnancy $\dagger$} \\
\hline No & 146 & 16 & 35 & $36 \cdot 1$ & 41 & $19 \cdot 4$ & 70 & $13 \cdot 7$ & 0.001 \\
\hline Yes & 673 & 74 & 62 & 63.9 & 170 & 80.6 & 441 & $86 \cdot 3$ & \\
\hline Gestational age during vitamin $D$ measurementł & & & & & & & & & 0.545 \\
\hline Median & \multirow{2}{*}{\multicolumn{2}{|c|}{$\begin{array}{c}26 \cdot 8 \\
26 \cdot 4-27 \cdot 6\end{array}$}} & \multirow{2}{*}{\multicolumn{2}{|c|}{$\begin{array}{c}27 \cdot 0 \\
26 \cdot 4-27 \cdot 7\end{array}$}} & \multirow{3}{*}{\multicolumn{2}{|c|}{$\begin{array}{c}26 \cdot 8 \\
26 \cdot 4-27.6\end{array}$}} & \multirow{2}{*}{\multicolumn{2}{|c|}{$\begin{array}{c}26 \cdot 9 \\
26 \cdot 4-27 \cdot 6\end{array}$}} & \\
\hline IQR & & & & & & & & & \\
\hline \multicolumn{8}{|l|}{ Infant characteristics } & & \\
\hline Birth weight $(\mathrm{g})$ & $3105 \cdot 0$ & $452 \cdot 6$ & 3143.0 & 414.8 & $3116 \cdot 8$ & $482 \cdot 4$ & 3091.5 & $447 \cdot 2$ & 0.474 \\
\hline Birth length $(\mathrm{cm})$ & 48.7 & $2 \cdot 3$ & 49.0 & $2 \cdot 1$ & 48.8 & $2 \cdot 3$ & 48.6 & $2 \cdot 3$ & 0.155 \\
\hline Birth order & & & & & & & & & 0.543 \\
\hline First child & 396 & 43.5 & 47 & $39 \cdot 2$ & 109 & $45 \cdot 2$ & 240 & 43.7 & \\
\hline Not first child & 514 & 56.5 & 73 & $60 \cdot 8$ & 132 & 54.8 & 309 & $56 \cdot 3$ & \\
\hline Infant sex & & & & & & & & & 0.221 \\
\hline Female & 436 & 47.9 & 56 & $46 \cdot 7$ & 127 & $52 \cdot 7$ & 253 & $46 \cdot 1$ & \\
\hline Male & 474 & 52.1 & 64 & $53 \cdot 3$ & 114 & $47 \cdot 3$ & 296 & 53.9 & \\
\hline SGA & & & & & & & & & 0.789 \\
\hline No & 827 & 90.9 & 110 & $91 \cdot 7$ & 221 & 91.7 & 496 & $90 \cdot 3$ & \\
\hline Yes & 83 & 9.1 & 10 & $8 \cdot 3$ & 20 & 8.3 & 53 & $9 \cdot 7$ & \\
\hline Pre-term & & & & & & & & & 0.919 \\
\hline Female & 840 & $92 \cdot 3$ & 111 & 92.5 & 221 & 91.7 & 508 & 92.5 & \\
\hline Male & 70 & $7 \cdot 7$ & 9 & 7.5 & 20 & 8.3 & 41 & 7.5 & \\
\hline
\end{tabular}

SGA, small for gestational age.

* Missing values imputed for covariates include the following: education (twelve imputed as post-secondary); smoked regularly before pregnancy (three imputed as no); maternal BMI (sixteen imputed as median=25.36). † $n 91$ had missing data. 
Table 2. Association of maternal vitamin $D$ status in pregnancy (independent variable) with small for gestational age (SGA) and pre-term risk (dependent variables) $(n 910)^{\star}$

(Odds ratios and $95 \%$ confidence intervals)

\begin{tabular}{|c|c|c|c|c|c|c|}
\hline & \multicolumn{2}{|c|}{$\begin{array}{l}\text { Vitamin D deficiency } \\
(<50 \mathrm{nmol} / /)(n 120)\end{array}$} & \multicolumn{2}{|c|}{$\begin{array}{c}\text { Vitamin D insufficiency } \\
(\geq 50 \text { and }<75 \mathrm{nmol} / \mathrm{l})(\text { ( } 241)\end{array}$} & \multicolumn{2}{|c|}{$\begin{array}{l}\text { Vitamin D sufficiency } \\
(>75 \mathrm{nmol} / /)(n 549)\end{array}$} \\
\hline & OR & $95 \% \mathrm{Cl}$ & OR & $95 \% \mathrm{Cl}$ & OR & $95 \% \mathrm{Cl}$ \\
\hline SGA† & 1.00 & $0.56,1.79$ & 1.08 & $0.50,2.34$ & & \\
\hline Pre-term† & $1 \cdot 16$ & $0 \cdot 64,2 \cdot 11$ & 1.06 & $0.48,2.38$ & & \\
\hline
\end{tabular}

Ref., referent values.

* Adjusted for maternal ethnicity, education, smoking during pregnancy, age, pregnancy BMI, total maternal energy intake, infant birth order and infant sex.

† No statistically significant differences were seen between maternal vitamin D deficiency status and SGA and pre-term outcomes.

or insufficient $25(\mathrm{OH}) \mathrm{D}$ levels after the adjustment for potential confounders (Table 2).

Tables 3 and 4 show that the weight, length (WAZ and LAZ) (Table 3), head, abdominal and mid-arm circumferences (Table 3), BMIZ (Table 4) and skinfold measurements from the triceps and subscapular regions (Table 4) at birth of infants whose mothers were sufficient in vitamin $D$ were not significantly different from those whose mothers were insufficient or deficient in vitamin $\mathrm{D}$, upon adjustment for potential confounders.

Sensitivity analysis in the subset ( $n$ 399) showed comparable findings, indicating no significant associations between maternal vitamin D status and infant weight and length measures, head, abdominal and mid-arm circumferences, BMI and skinfold measurements at birth $(P>0.006)$ (online Supplementary Tables S3-S6).

Postnatal growth outcomes from 3 to 24 months of age. After adjustment for potential confounding factors, the postnatal growth outcomes of weight and length (WAZ and LAZ) (Table 3), head, abdominal and mid-arm circumferences (Table 3), and BMIZ (Table 4) in infants whose mothers were sufficient in vitamin D were not significantly different from infants whose mothers were insufficient or deficient in vitamin D. This was seen consistently from 3 to 24 months of age. Similarly, no associations were observed with adiposity outcomes such as BMIZ (Table 4) and infant skinfold measurements at 18 and 24 months of age (Table 4).

Results from the conditional change analysis in LAZ, WAZ (online Supplementary Table S7) and BMIZ (online Supplementary Table s8) showed that length and weight gain in infants whose mothers were sufficient in vitamin D were not significantly different from those whose mothers were insufficient or deficient in vitamin D.

Sensitivity analysis of the subset ( $n$ 399) with complete postnatal growth data gave largely similar findings, such that no significant associations were observed between maternal vitamin D status and infant weight and length measures, head, abdominal and mid-arm circumferences, BMI and skinfold measurements. However, we observed a non-statistically significant trend between maternal vitamin D deficiency and shorter infant length, lower weight (LAZ and WAZ), smaller BMIZ and smaller abdominal and mid-arm circumferences, as reflected by the negative $\beta$-coefficient values observed at all time points from 3 up to 24 months of age $(P>0.006)$ (online Supplementary Tables S3-S5). In addition, we found a statistically significant association between maternal vitamin $\mathrm{D}$ insufficiency and lower infant subscapular skinfold at 24 months of age $(\beta=-0.64 ; 95 \%$ CI $-1.07,-0.22 ; P=0.003)$ (online Supplementary Table S6).

Findings from analyses conducted using maternal plasma vitamin $\mathrm{D}$ concentrations as a continuous measure also did not show statistically significant associations with growth and adiposity outcomes (data not shown). Results for the unadjusted analysis are available in the online Supplementary Tables S9-S11.

\section{Discussion}

Findings from this longitudinal cohort suggest that maternal vitamin D deficiency (25(OH)D levels $<50 \mathrm{nmol} / \mathrm{l})$ and insufficiency ( $\geq 50$ and $<75 \mathrm{nmol} / \mathrm{l}$ ) during the mid-late trimester of pregnancy are not associated with birth outcomes, postnatal growth and adiposity outcomes in the first 2 years of life in a multi-ethnic Asian population.

Similar to other prospective cohort studies ${ }^{(16,17)}$ and a multimicronutrient clinical trial ${ }^{(29)}$, which used $<50 \mathrm{nmol} / 1$ to define vitamin $\mathrm{D}$ deficiency, our study showed a null association between maternal vitamin D deficiency and risk of SGA and pre-term birth. In contrast, observational studies reporting significant associations with risk of SGA and pre-term births ${ }^{(5,6,8,9)}$ have higher prevalence of deficiency defined by a lower vitamin D deficiency cut-off of range: $30-37.5 \mathrm{nmol} / \mathrm{l}$.

Our cohort also showed null associations between maternal vitamin D status and birth measures such as birth weight and length. This observation agrees with several other cohort studies $^{(13-15,17)}$, and we observed that studies reporting null findings generally defined higher cut-offs $(37 \cdot 5-80 \mathrm{nmol} / \mathrm{l})$ for vitamin $\mathrm{D}$ deficiency ${ }^{(13-15,17)}$, with vitamin $\mathrm{D}$ prevalence that ranged from 20 to $66 \%$. Cohort studies ${ }^{(5,11,30)}$ and crosssectional studies ${ }^{(6,12,31)}$ have reported maternal vitamin D deficiency to be associated with poorer weight, length, BMI or percentile growth at birth. It was notable that these cohort studies tended to use lower vitamin D deficiency cut-offs ${ }^{(5,11,30)}$ (range: $29 \cdot 9-37.5 \mathrm{nmol} / \mathrm{l})$. The cross-sectional studies, however, were limited by small sample sizes ${ }^{(6,12)}(n 56-70)$, or had used dietary data to assess maternal $25(\mathrm{OH}) \mathrm{D}$ levels ${ }^{(31)}$.

Other anthropometric measures at birth, including infant head, abdominal and mid-arm circumferences, in our study also revealed null associations with maternal vitamin D status. These findings are in contrast with two other cohort studies 
Table 3. Association of maternal vitamin D status in pregnancy (independent variable) with infant weight-for-age $z$-scores, length-for-age $z$-scores and head, abdominal and mid-arm circumferences from $0-24$ months (dependent variables) $(n 807)^{*}$

( $\beta$-Coefficients and $95 \%$ confidence intervals)

\begin{tabular}{|c|c|c|c|c|c|c|c|c|}
\hline & \multicolumn{3}{|c|}{ Vitamin $D$ deficiency $(<50 \mathrm{nmol} / \mathrm{l})$} & \multicolumn{3}{|c|}{ Vitamin D insufficiency $(\geq 50$ and $<75 \mathrm{nmol} / \mathrm{l})$} & \multicolumn{2}{|c|}{ Vitamin D sufficiency $(>75 \mathrm{nmol} / \mathrm{l})$} \\
\hline & $n$ & $\beta$ & $95 \% \mathrm{Cl}$ & $n$ & $\beta$ & $95 \% \mathrm{Cl}$ & $n$ & $\beta$ \\
\hline \multicolumn{9}{|c|}{ Weight-for-age $z$-score $\dagger$} \\
\hline 0 months & 109 & 0.09 & $-0.07,0.26$ & 215 & 0.09 & $-0.03,0.22$ & 483 & Ref. \\
\hline 3 months & 98 & -0.02 & $-0.23,0.19$ & 203 & -0.05 & $-0 \cdot 21,0 \cdot 11$ & 458 & Ref. \\
\hline 6 months & 86 & 0.00 & $-0.23,0.23$ & 195 & -0.01 & $-0.18,0.16$ & 448 & Ref. \\
\hline 9 months & 85 & 0.00 & $-0.23,0.23$ & 181 & -0.01 & $-0.19,0.16$ & 428 & Ref. \\
\hline 12 months & 89 & 0.01 & $-0.22,0.24$ & 186 & 0.07 & $-0.10,0.24$ & 436 & Ref. \\
\hline 15 months & 86 & -0.02 & $-0.25,0.21$ & 190 & 0.06 & $-0.11,0.23$ & 442 & Ref. \\
\hline 18 months & 86 & -0.06 & $-0.30,0.18$ & 184 & 0.11 & $-0.07,0.29$ & 414 & Ref. \\
\hline 24 months & 79 & 0.00 & $-0.27,0.26$ & 189 & 0.02 & $-0.16,0.21$ & 418 & Ref. \\
\hline \multicolumn{9}{|c|}{ Length-for-age $z$-score $\dagger$} \\
\hline 0 months & 109 & 0.29 & $0.07,0.51$ & 213 & 0.21 & $0.04,0.38$ & 483 & Ref. \\
\hline 3 months & 98 & -0.02 & $-0.26,0.22$ & 203 & 0.01 & $-0.17,0.19$ & 458 & Ref. \\
\hline 6 months & 87 & -0.16 & $-0.43,0.12$ & 196 & 0.05 & $-0.15,0.25$ & 450 & Ref. \\
\hline 9 months & 85 & -0.05 & $-0.33,0.22$ & 181 & 0.02 & $-0.19,0.22$ & 428 & Ref. \\
\hline 12 months & 89 & 0.10 & $-0.17,0.38$ & 188 & 0.06 & $-0.15,0.26$ & 437 & Ref. \\
\hline 15 months & 86 & -0.02 & $-0.29,0.25$ & 189 & -0.03 & $-0.23,0.17$ & 437 & Ref. \\
\hline 18 months & 75 & -0.13 & $-0.41,0.16$ & 164 & 0.21 & $0.00,0.42$ & 357 & Ref. \\
\hline 24 months $\ddagger$ & 70 & $-0 \cdot 10$ & $-0.39,0.19$ & 168 & 0.04 & $-0.16,0.25$ & 371 & Ref. \\
\hline \multicolumn{9}{|c|}{ Head circumference $(\mathrm{cm}) \dagger$} \\
\hline 0 months & 103 & 0.06 & $-0.21,0.34$ & 206 & 0.07 & $-0.14,0.28$ & 469 & Ref. \\
\hline 3 months & 97 & -0.04 & $-0.30,0.23$ & 203 & 0.04 & $-0.16,0.24$ & 458 & Ref. \\
\hline 6 months & 87 & 0.11 & $-0.20,0.41$ & 193 & 0.09 & $-0.14,0.31$ & 443 & Ref. \\
\hline 9 months & 85 & 0.13 & $-0.20,0.45$ & 180 & -0.07 & $-0.32,0.17$ & 428 & Ref. \\
\hline 12 months & 89 & 0.08 & $-0.24,0.40$ & 188 & 0.00 & $-0.23,0.24$ & 436 & Ref. \\
\hline 15 months & 86 & $0 \cdot 10$ & $-0.23,0.43$ & 190 & -0.01 & $-0.25,0.23$ & 443 & Ref. \\
\hline 18 months & 82 & 0.09 & $-0.29,0.46$ & 183 & 0.05 & $-0.22,0.33$ & 410 & Ref. \\
\hline 24 months & 80 & -0.23 & $-0.59,0.13$ & 186 & 0.03 & $-0.23,0.30$ & 410 & Ref. \\
\hline \multicolumn{9}{|c|}{ Abdominal circumference $(\mathrm{cm}) \dagger$} \\
\hline 0 months & 102 & 0.08 & $-0.43,0.60$ & 206 & $0 \cdot 10$ & $-0.30,0.49$ & 469 & Ref. \\
\hline 3 months & 98 & 0.02 & $-0.65,0.68$ & 202 & 0.11 & $-0.39,0.61$ & 457 & Ref. \\
\hline 6 months & 87 & 0.34 & $-0.40,1.08$ & 194 & $0 \cdot 16$ & $-0.39,0.70$ & 446 & Ref. \\
\hline 9 months & 85 & 0.11 & $-0.66,0.87$ & 180 & 0.17 & $-0.40,0.74$ & 427 & Ref. \\
\hline 12 months & 89 & 0.17 & $-0.59,0.93$ & 188 & -0.10 & $-0.66,0.47$ & 437 & Ref. \\
\hline 15 months & 86 & 0.00 & $-0.74,0.74$ & 188 & 0.12 & $-0.42,0.67$ & 443 & Ref. \\
\hline 18 months & 80 & -0.01 & $-0.81,0.80$ & 176 & 0.22 & $-0.38,0.81$ & 394 & Ref. \\
\hline 24 months & 78 & -0.18 & $-1.06,0.71$ & 178 & 0.17 & $-0.47,0.81$ & 402 & Ref. \\
\hline \multicolumn{9}{|c|}{ Mid-arm circumference $(\mathrm{cm}) \dagger$} \\
\hline 0 months & 102 & 0.04 & $-0.15,0.24$ & 206 & -0.07 & $-0.22,0.08$ & 470 & Ref. \\
\hline 3 months & 98 & 0.06 & $-0.21,0.34$ & 202 & 0.02 & $-0.19,0.22$ & 456 & Ref. \\
\hline 6 months & 86 & 0.05 & $-0.25,0.34$ & 195 & 0.03 & $-0.18,0.25$ & 447 & Ref. \\
\hline 9 months & 85 & -0.02 & $-0.32,0.28$ & 180 & -0.06 & $-0.29,0.16$ & 427 & Ref. \\
\hline 12 months & 89 & 0.09 & $-0.19,0.37$ & 188 & -0.05 & $-0.25,0.16$ & 437 & Ref. \\
\hline 15 months & 86 & 0.11 & $-0.19,0.40$ & 190 & 0.02 & $-0.20,0.23$ & 443 & Ref. \\
\hline 18 months & 83 & -0.06 & $-0.37,0.25$ & 181 & 0.04 & $-0.19,0.27$ & 403 & Ref. \\
\hline 24 months & 78 & -0.04 & $-0.38,0.30$ & 187 & -0.04 & $-0.29,0.20$ & 404 & Ref. \\
\hline
\end{tabular}

Ref. referent values.

* Adjusted for maternal ethnicity, education, smoking during pregnancy, age, pregnancy BMI, total maternal energy intake, infant birth order and infant sex.

$\dagger$ No statistically significant differences were seen between maternal vitamin D deficiency status and weight-for-age, length-for-age $z$-score and head circumference, abdominal circumference and mid-arm circumference.

$\ddagger$ No statistically significant differences were seen between maternal vitamin $D$ deficiency status and height-for-age $z$-scores were measured only at 24 months. 
that found inverse relationships between maternal vitamin D concentrations and infant head circumference at birth, even though they had a high population median vitamin $D$ concentration of $73.38 \mathrm{nmol} / \mathrm{l}^{(16)}$ and mean vitamin $\mathrm{D}$ concentration of $70.5 \mathrm{nmol} / \mathrm{l}^{(17)}$. In a study that defined a lower vitamin D deficiency cut-off ( $<37.5 \mathrm{nmol} / \mathrm{l})$, maternal vitamin D deficiency was reported to be related to lower infant head circumference at birth ${ }^{(30)}$. This is, however, in line with supplementation trials conducted in populations of low levels of maternal vitamin D $($ mean $=31.7-39.7 \mathrm{nmol} / \mathrm{l})$, where most have reported the significant effects ${ }^{(29,32,33)}$ of maternal supplementation on increasing infant head circumference. Our findings related to mid-arm circumference were supported by one study reporting similar null associations between maternal vitamin D deficiency $(<30 \mathrm{nmol} / \mathrm{l})$ and mid-arm circumference at 9 months of age ${ }^{(14)}$, whereas data on infant abdominal circumference are lacking.

In our study, postnatal growth outcomes were generally not affected by maternal deficiency in vitamin D during pregnancy. With regard to weight outcomes, our results were similar to studies by van Eijsden et al. and Prentice et al., where no significant associations were found of maternal 25(OH)D levels (mean $=65.9 \mathrm{nmol} / \mathrm{l})$ with weight in children between ages $4-5$ years $^{(34)}$ and vitamin D deficiency $(<80 \mathrm{nnol} / \mathrm{l})$ with weight in infants at 2, 3 and 13 weeks postpartum, respectively ${ }^{(15)}$ In contrast, a study by Eckhardt et al. ${ }^{(11)}$ has reported associations of maternal vitamin D deficiency $(<30 \mathrm{nmol} / \mathrm{l})$ with greater infant weight at 6 and 9 months of age. With respect to length outcomes, our study showed similar results as studies by van Eijsden et $a l .{ }^{(34)}$ and Prentice et $a l^{(15)}$, where no associations were observed,. In contrast, Leffelaar et al. ${ }^{(5)}$ reported maternal vitamin $\mathrm{D}$ deficiency to be associated with decreased infant length at months 9 and 12, whereas Eckhardt et al. ${ }^{(11)}$ found lower infant length at 1 month but higher length at 12 months. Finally, with respect to head circumference outcomes, Prentice et $a l .{ }^{(15)}$ again reported no significant associations with maternal vitamin D deficiency. However, a study by Gale et al. ${ }^{(14)}$ that examined postnatal growth at 9 months of age and subsequently at 9 years of age did not show any association between maternal vitamin D deficiency $(<30 \mathrm{nmol} / \mathrm{l})$ and most growth outcomes (weight, length, height and mid-arm circumference), with the exception of a significant association with larger head circumference at 9 years. Despite the inconclusiveness of the studies surrounding postnatal growth, in general, studies with lower vitamin D deficiency cut-offs $<30 \mathrm{nmol} / 1$ found significant associations in their studies ${ }^{(5,11,14)}$ but studies with higher cutoffs $(<80 \mathrm{nmol} / \mathrm{l})$ did not ${ }^{(15,34)}$.

With regard to the associations between vitamin D deficiency and adiposity outcomes, we found no associations between maternal vitamin $\mathrm{D}$ and our birth and postnatal adiposity outcomes (BMI and skinfold thickness). Studies involving adiposity outcomes such as BMI are lacking and conflicting. Gale et $a l^{(14)}$ reported no significant associations between vitamin D deficiency (deficiency defined as $<30 \mathrm{nmol} / \mathrm{l}$ ) and infant BMI at 9 years of age, whereas Lee et al. ${ }^{(18)}$ reported vitamin D concentrations $($ mean $=48.9-75.8 \mathrm{nmol} / \mathrm{l})$ to be inversely associated with BMI in pre-adolescents aged 7-9 years. In relation to skinfold thickness, Farrant et al. ${ }^{(13)}$ reported no significant associations between maternal vitamin D deficiency during pregnancy and infant triceps and subscapular skinfold measurements at birth, which is consistent with our findings. Existing studies have not investigated maternal vitamin D with infant skinfold measurements following birth except for the preadolescent study by Lee et $a l{ }^{(18)}$, which has reported an inverse association between vitamin D concentrations and triceps skinfold thickness. Overall, there is still a lack of studies on maternal vitamin D and adiposity outcomes in early childhood, which requires further investigation.

Null associations were also seen with our additional analysis of conditional $z$-score change in length, weight and BMI, which shows that the length gain and weight gain from 3 up to 24 months were not significantly different between vitamin D-deficient mothers and those who were sufficient. None of the studies discussed has conducted similar analyses, which make comparisons difficult. However, this form of analysis provides an advantage to our study as this analytical approach enables us to examine the influence of maternal vitamin $\mathrm{D}$ on growth and adiposity outcomes at later time points, independent of growth and adiposity outcomes at earlier time points ${ }^{(35)}$

In the sensitivity analyses of a subset of our cohort, we did note a non-significant trend of maternal vitamin D deficiency being related to slower infant linear growth, smaller abdominal and mid-arm circumferences, and lower infant BMI in the first 24 months of life. In addition, some evidence indicated that maternal vitamin D deficiency was significantly associated with reduced infant subscapular skinfold at 24 months. This lends support to general evidence that severe maternal vitamin D deficiency can lead to poorer infant growth $^{(5,8,11,12)}$

The lack of significant associations in our study may be a consequence of lower prevalence of vitamin $\mathrm{D}$ deficiency in our population compared with cohorts in other countries. In our cohort, $13.2 \%$ were vitamin $\mathrm{D}$ deficient (defined as $<50.0 \mathrm{nmol} / \mathrm{l})$, whereas only $1.6 \%$ of the cohort had severe vitamin D deficiency (defined as of $<30 \cdot 0 \mathrm{nmol} / \mathrm{l}$ ).

\section{Strengths and limitations}

To our knowledge, this is the first prospective cohort study to have comprehensively investigated the associations between maternal vitamin D during pregnancy and infant birth and growth outcomes with 3-month follow-up intervals till the age of 2 years.

The strengths of this study are the prospective study design, short intervals between follow-up sessions to track infant growth from birth and the range of outcomes that have been measured including birth outcomes, growth outcomes and adiposity outcomes. Only singleton term-born infants were included in our analysis on infant growth, whereas pre-term and multiple birth infants were excluded because of differences in growth trajectories ${ }^{(36)}$. Furthermore, the findings of our study are generalisable to other neighbouring Southeast Asian countries such as Indonesia $\left(6^{\circ} \mathrm{S}\right)$ and Malaysia $\left(2^{\circ} \mathrm{N}\right)$, which lie at the closest latitude to Singapore and have reported vitamin D 
Table 4. Association of maternal vitamin $D$ status in pregnancy (independent variable) with infant BMI $z$-score from 0-24 months and infant skinfold measurements at 0,18 and 24 months (dependent variable) $(n 807)^{*}$

( $\beta$-Coefficients and $95 \%$ confidence intervals)

\begin{tabular}{|c|c|c|c|c|c|c|c|c|}
\hline & \multicolumn{3}{|c|}{ Vitamin D deficiency $(<50 \mathrm{nmol} / \mathrm{l})$} & \multicolumn{3}{|c|}{ Vitamin D insufficiency $(\geq 50$ and $<75 \mathrm{nmol} / \mathrm{l})$} & \multicolumn{2}{|c|}{ Vitamin D sufficiency $(>75 \mathrm{nmol} / \mathrm{l})$} \\
\hline & $n$ & $\beta$ & $95 \% \mathrm{Cl}$ & $n$ & $\beta$ & $95 \% \mathrm{Cl}$ & $n$ & $\beta$ \\
\hline \multicolumn{9}{|c|}{ BMI-for-age $z$-score $\dagger$} \\
\hline 0 months & 109 & -0.09 & $-0.29,0.12$ & 213 & -0.02 & $-0.18,0.13$ & 483 & Ref. \\
\hline 3 months & 98 & -0.01 & $-0.25,0.22$ & 203 & -0.07 & $-0.25,0.10$ & 458 & Ref. \\
\hline 6 months & 86 & 0.11 & $-0.15,0.37$ & 195 & -0.07 & $-0.26,0.12$ & 448 & Ref. \\
\hline 9 months & 85 & 0.04 & $-0.22,0.29$ & 181 & -0.04 & $-0.23,0.15$ & 428 & Ref. \\
\hline 12 months & 89 & -0.07 & $-0.31,0.17$ & 186 & 0.04 & $-0.14,0.22$ & 436 & Ref. \\
\hline 15 months & 86 & -0.02 & $-0.27,0.22$ & 189 & 0.09 & $-0.09,0.27$ & 437 & Ref. \\
\hline 18 months & 75 & 0.01 & $-0.26,0.27$ & 163 & 0.02 & $-0.18,0.21$ & 356 & Ref. \\
\hline 24 months & 70 & 0.02 & $-0.27,0.30$ & 168 & -0.04 & $-0.25,0.16$ & 371 & Ref. \\
\hline \multicolumn{9}{|c|}{ Triceps skinfold $\ddagger$} \\
\hline 0 months & 103 & 0.12 & $-0.15,0.39$ & 206 & -0.03 & $-0.24,0.18$ & 470 & Ref. \\
\hline 18 months & 78 & 0.37 & $-0.08,0.81$ & 166 & 0.02 & $-0.32,0.35$ & 365 & Ref. \\
\hline 24 months & 72 & -0.02 & $-0.52,0.49$ & 171 & -0.14 & $-0.51,0.22$ & 378 & Ref. \\
\hline \multicolumn{9}{|c|}{ Biceps skinfold $\neq$} \\
\hline 18 months & 76 & 0.29 & $-0.13,0.71$ & 159 & -0.02 & $-0.34,0.29$ & 341 & Ref. \\
\hline 24 months & 69 & -0.05 & $-0.45,0.35$ & 167 & -0.01 & $-0.30,0.28$ & 371 & Ref. \\
\hline \multicolumn{9}{|c|}{ Subscapular skinfoldł } \\
\hline 0 months & 103 & -0.07 & $-0.32,0.18$ & 206 & 0.00 & $-0.19,0.19$ & 469 & Ref. \\
\hline 18 months & 69 & 0.19 & $-0.18,0.57$ & 155 & -0.10 & $-0.37,0.18$ & 341 & Ref. \\
\hline 24 months & 74 & 0.19 & $-0.24,0.61$ & 178 & -0.30 & $-0.60,0.00$ & 385 & Ref. \\
\hline
\end{tabular}

Ref. referent values.

* Adjusted for maternal ethnicity, education, smoking during pregnancy, age, pregnancy BMI, total maternal energy intake and infant birth order.

$\dagger$ No statistically significant differences were seen between maternal vitamin D deficiency status and BMI-for-age $z$-score.

$\ddagger$ No statistically significant differences were seen between maternal vitamin D deficiency status and triceps skinfold, biceps skinfold and subscapular skinfold.

inadequacy in their pregnant women. Despite these countries receiving sunlight all year round, similar to Singapore, cultural practices within the different ethnic groups could contribute to the difference in the $25(\mathrm{OH}) \mathrm{D}$ levels. For example, the common custom is for most Malay or Muslim women to cover their skin, which could lead to lower levels of $25(\mathrm{OH}) \mathrm{D}$ levels. In addition, both Malay and Indian ethnic groups have darker skin pigmentation than Chinese, and would require longer duration of sunlight exposure to synthesise vitamin $\mathrm{D}_{3}$ contributing to lower levels of vitamin D in these ethnic groups ${ }^{(37)}$. In general, the better-educated mothers in Singapore could also be taking steps to prevent skin cancer by using sun protection, umbrellas or wearing clothing that protect them from the sun, such as long-sleeved shirts and jeans.

Our study also has certain limitations that need to be addressed. First, our analysis was performed only on data of women whose vitamin D status had been measured and where growth data of the infants were available. Owing to the intensive follow-up sessions, the number of subjects decreased at later time points, resulting in missing data at later time points, which may have affected the analytical power of our analyses.

Second, maternal plasma vitamin D concentration was only assessed once in late pregnancy, because the mobilisation of vitamin D from maternal stores for fetal development is greatest during this period ${ }^{(4)}$. However, assessment of plasma vitamin D concentrations at different periods of pregnancy ${ }^{(30)}$ could provide useful insight into how the mobilisation of maternal vitamin D stores during different pregnancy periods may affect infants differently.
Third, we were unable to directly compare our results with studies that have used vitamin $\mathrm{D}$ cut-offs for deficiency based on the IOM guidelines $(<30 \mathrm{nmol} / \mathrm{l})$. This is because only $1.6 \%$ of the mothers in our cohort had severe vitamin D deficiency $(<30 \cdot 0 \mathrm{nmol} / \mathrm{l})$, which did not provide sufficient power for analyses.

Finally, due to the observational nature of our study, there is a possibility of residual confounding arising from other factors such as infant UV-B exposure or infant vitamin D supplementation. The use of infant vitamin D supplementation was not taken into account in our analysis due to the low prevalence of supplementation within our cohort. On the basis of data from administered questionnaires at the clinic visits, only $6.92 \%$ of the infants in this study cohort were reported to have taken supplements containing vitamin D up to 12 months of age (data not shown), whereas $74.0 \%$ of the mothers were taking supplements containing vitamin D during pregnancy (Table 1).

A major drawback in the existing literature of vitamin D studies is the inconsistent cut-offs used to define vitamin D deficiency. In general, it was observed that studies that used lower cut-offs tended to reported significant associations between maternal vitamin D and infant outcomes ${ }^{(5,8,11)}$, whereas studies that used higher cut-offs tended to report nonsignificant associations ${ }^{(13,15-17)}$. The inconsistency observed in past studies makes it difficult to draw reliable conclusions when these results are compared. Furthermore, and to the best of our knowledge, there are no studies looking at dose-response relationships across the vitamin $\mathrm{D}$ range that directly address this issue. 


\section{Conclusion}

We did not find an association between maternal vitamin D deficiency and infant birth outcomes or postnatal growth and adiposity measures in our cohort. We think that this can be explained by the low prevalence of severe vitamin D deficiency within this population. Future studies using standardised and established cut-offs defining vitamin D deficiency will enable findings across studies to be more comparable.

\section{Acknowledgements}

This study acknowledges the contribution of the rest of the GUSTO study group, which includes the following: Pratibha Agarwal, Arijit Biswas, Choon Looi Bong, Birit F. P. Broekman, Shirong Cai, Jerry Kok Yen Chan, Yiong Huak Chan, Cornelia Yin Ing Chee, Helen Y. H. Chen, Yin Bun Cheung, Audrey Chia, Amutha Chinnadurai, Chai Kiat Chng, Shang Chee Chong, Mei Chien Chua, Chun Ming Ding, Eric Andrew Finkelstein, Doris Fok, Marielle V. Fortier, Anne Eng Neo Goh, Yam Thiam Daniel Goh, Joshua J. Gooley, Wee Meng Han, Mark Hanson, Christiani Jeyakumar Henry, Joanna D. Holbrook, Chin-Ying Hsu, Hazel Inskip, Jeevesh Kapur, Kenneth Kwek, Ivy Yee-Man Lau, Bee Wah Lee, Yung Seng Lee, Ngee Lek, Sok Bee Lim, Yen-Ling Low, Iliana Magiati, Lourdes Mary Daniel, Michael Meaney, Cheryl Ngo, Krishnamoorthy Naiduvaje, Wei Wei Pang, Anqi Qiu, Boon Long Quah, Victor Samuel Rajadurai, Mary Rauff, Salome A. Rebello, Jenny L. Richmond, Anne Rifkin-Graboi, Seang-Mei Saw, Lynette Pei-Chi Shek, Allan Sheppard, Borys Shuter, Leher Singh, Shu-E. Soh, Walter Stunkel, Lin Lin Su, Kok Hian Tan, Oon Hoe Teoh, Hugo P. S. van Bever, Inez Bik Yun Wong, P. C. Wong, George Seow Heong Yeo.

This research is supported by the Singapore National Research Foundation under its Translational and Clinical Research (TCR) Flagship Programme and administered by the Singapore Ministry of Health's National Medical Research Council (NMRC), Singapore NMRC/TCR/004-NUS/2008; NMRC/TCR/012-NUHS/2014. Additional funding of the present study was provided by the Singapore Institute for Clinical Sciences, A*STAR and Nestec. Study sponsors were not involved in the design of the study, statistical analysis and results interpretation.

The contributions are as follows: S.-M. S., K. M. G., P. D. G., Y. S. C., F. Y. and Y. S. L. designed and led the GUSTO study. Y. L. O. and P. L. Q. both contributed equally to the statistical analysis and writing of the manuscript. I. M. A., R. M. v. D. and D. H. provided intellectual contribution to the write-up of the manuscript. P. L. Q. and M. F. F. C. were responsible for finalising the manuscript. M. T. T. carried out the data collection for the study. All authors were involved in all parts of the study and approved the final version of the manuscript.

P. D. G., K. M. G. and Y. S. C. have received reimbursement for speaking at conferences sponsored by companies selling nutritional products. These authors are part of an academic consortium that has received research funding from Abbot Nutrition, Nestec and Danone. None of the other authors report any potential conflicts of interest.

\section{Supplementary material}

For supplementary material/s referred to in this article, please visit http://dx.doi.org/10.1017/S0007114516000623

\section{References}

1. Barker DJ \& Clark PM (1997) Fetal undernutrition and disease in later life. Rev Reprod 2, 105-112.

2. Principi N, Bianchini S, Baggi E, et al. (2013) Implications of maternal vitamin D deficiency for the fetus, the neonate and the young infant. Eur J Nutr 52, 859-867.

3. Walsh JM, Kilbane M, McGowan CA, et al. (2013) Pregnancy in dark winters: implications for fetal bone growth? Fertil Steril 99, 206-211.

4. Weinert LS \& Silveiro SP (2015) Maternal-fetal impact of vitamin D deficiency: a critical review. Matern Child Health J 19 , 94-101.

5. Leffelaar ER, Vrijkotte TG \& van Eijsden M (2010) Maternal early pregnancy vitamin $\mathrm{D}$ status in relation to fetal and neonatal growth: results of the multi-ethnic Amsterdam born children and their development cohort. BrJ Nutr 104, 108-117.

6. Robinson CJ, Wagner CL, Hollis BW, et al. (2011) Maternal vitamin $\mathrm{D}$ and fetal growth in early-onset severe preeclampsia. Am J Obstet Gynecol 204, 556.e551-e 554.

7. Finkelstein JL, Mehta S, Duggan C, et al. (2012) Maternal vitamin D status and child morbidity, anemia, and growth in human immunodeficiency virus-exposed children in Tanzania. Pediatr Infect Dis J 31, 171-175.

8. Gernand AD, Simhan HN, Caritis S, et al. (2014) Maternal vitamin D status and small-for-gestational-age offspring in women at high risk for preeclampsia. Obstet Gynecol 123, $40-48$.

9. Bodnar LM, Catov JM, Zmuda JM, et al. (2010) Maternal serum 25-hydroxyvitamin D concentrations are associated with small-for-gestational age births in white women. J Nutr 140, 999-1006.

10. De-Regil LM, Palacios C, Ansary A, et al. (2012) Vitamin D supplementation for women during pregnancy. The Cochrane Database of Systematic Reviews 2012, issue 2, CD008873.

11. Eckhardt CL, Gernand AD, Roth DE, et al. (2015) Maternal vitamin D status and infant anthropometry in a US multi-centre cohort study. Ann Hum Biol 42, 215-222.

12. Song SJ, Si S, Liu J, et al. (2013) Vitamin D status in Chinese pregnant women and their newborns in Beijing and their relationships to birth size. Public Health Nutr 16, 687-692.

13. Farrant HJ, Krishnaveni GV, Hill JC, et al. (2009) Vitamin D insufficiency is common in Indian mothers but is not associated with gestational diabetes or variation in newborn size. Eur J Clin Nutr 63, 646-652.

14. Gale CR, Robinson SM, Harvey NC, et al. (2008) Maternal vitamin D status during pregnancy and child outcomes. Eur J Clin Nutr 62, 68-77.

15. Prentice A, Jarjou LM, Goldberg GR, et al. (2009) Maternal plasma 25-hydroxyvitamin D concentration and birthweight, growth and bone mineral accretion of Gambian infants. Acta Paediatr 98, 1360-1362.

16. Rodriguez A, Garcia-Esteban R, Basterretxea M, et al. (2015) Associations of maternal circulating 25-hydroxyvitamin $\mathrm{D}_{3}$ concentration with pregnancy and birth outcomes. BJOG 122, 1695-1704.

17. Hanieh S, Ha TT, Simpson JA, et al. (2014) Maternal vitamin D status and infant outcomes in rural Vietnam: a prospective cohort study. PLOS ONE 9, e99005. 
18. Lee HA, Kim YJ, Lee $\mathrm{H}$, et al. (2013) Association of vitamin $\mathrm{D}$ concentrations with adiposity indices among preadolescent children in Korea. J Pediatr Endocrinol Metab 26, 849-854.

19. Morales E, Rodriguez A, Valvi D, et al. (2015) Deficit of vitamin $\mathrm{D}$ in pregnancy and growth and overweight in the offspring. Int J Obes (Lond) 39, 61-68.

20. Crozier SR, Harvey NC, Inskip HM, et al. (2012) Maternal vitamin $\mathrm{D}$ status in pregnancy is associated with adiposity in the offspring: findings from the Southampton Women's Survey. Am J Clin Nutr 96, 57-63.

21. Soh SE, Tint MT, Gluckman PD, et al. (2014) Cohort profile: Growing Up in Singapore Towards healthy Outcomes (GUSTO) birth cohort study. Int J Epidemiol 43, 1401-1409.

22. Goh SK (2011) Birthweight percentiles by gestational age and maternal factors that affect birthweight in Singapore. Master of Science, National University of Singapore, Singapore.

23. Maunsell Z, Wright DJ \& Rainbow SJ (2005) Routine isotopedilution liquid chromatography-tandem mass spectrometry assay for simultaneous measurement of the 25-hydroxy metabolites of vitamins $\mathrm{D}_{2}$ and $\mathrm{D}_{3}$. Clin Chem 51, 1683-1690.

24. Hamilton CM, Strader LC, Pratt JG, et al. (2011) The PhenX Toolkit: get the most from your measures. Am J Epidemiol 174, 253-260.

25. Schaefer F, Georgi M, Zieger A, et al. (1994) Usefulness of bioelectric impedance and skinfold measurements in predicting fat-free mass derived from total body potassium in children. Pediatr Res 35, 617-624.

26. Holick MF, Binkley NC, Bischoff-Ferrari HA, et al. (2011) Evaluation, treatment, and prevention of vitamin D deficiency: an Endocrine Society clinical practice guideline. J Clin Endocrinol Metab 96, 1911-1930.

27. Bloem M (2007) The 2006 WHO child growth standards. BMJ 334, 705-706.
28. Corvalan C (2014) Unconditional or conditional change: does it matter? Growth charts for monitoring weight gain during pregnancy. Am J Clin Nutr 99, 245-246.

29. Brough L, Rees GA, Crawford MA, et al. (2010) Effect of multiple-micronutrient supplementation on maternal nutrient status, infant birth weight and gestational age at birth in a lowincome, multi-ethnic population. Br J Nutr 104, 437-445.

30. Gernand AD, Simhan HN, Klebanoff MA, et al. (2013) Maternal serum 25-hydroxyvitamin D and measures of newborn and placental weight in a U.S. multicenter cohort study. J Clin Endocrinol Metab 98, 398-404.

31. Sabour H, Hossein-Nezhad A, Maghbooli Z, et al. (2006) Relationship between pregnancy outcomes and maternal vitamin D and calcium intake: a cross-sectional study. Gynecol Endocrinol 22, 585-589.

32. Hashemipour S, Ziaee A, Javadi A, et al. (2014) Effect of treatment of vitamin D deficiency and insufficiency during pregnancy on fetal growth indices and maternal weight gain: a randomized clinical trial. Eur J Obstet Gynecol Reprod Biol 172, 15-19.

33. Kalra P, Das V, Agarwal A, et al. (2012) Effect of vitamin D supplementation during pregnancy on neonatal mineral homeostasis and anthropometry of the newborn and infant. Br J Nutr 108, 1052-1058.

34. van Eijsden M, Snijder MB, Brouwer I, et al. (2013) Maternal early-pregnancy vitamin D status in relation to linear growth at the age of 5-6 years: results of the ABCD cohort. Eur J Clin Nutr 67, 972-977.

35. Cole TJ (1995) Conditional reference charts to assess weight gain in British infants. Arch Dis Child 73, 8-16.

36. Sullivan MC, McGrath MM, Hawes K, et al. (2008) Growth trajectories of preterm infants: birth to 12 years. J Pediatr Health Care 22, 83-93.

37. Nimitphong H \& Holick MF (2013) Vitamin D status and sun exposure in southeast Asia. Dermatoendocrinol 5, 34-37. 